\title{
Stress singularities produced by fluid structure interactions
}

\author{
G. B. Sinclair ${ }^{1}$, X. Chi $^{2}$ \& T. I-P. Shih ${ }^{2}$ \\ ${ }^{1}$ Department of Mechanical Engineering, Louisiana State University, \\ USA \\ ${ }^{2}$ Department of Aerospace Engineering, Iowa State University, USA
}

\begin{abstract}
Essential for meaningful design for structural integrity is an appreciation of when stress singularities are present in structural configurations. While there is a rich literature devoted to the identification of such singular behavior in solid mechanics, to date there has been relatively little explicit identification of stress singularities caused by fluid flows. In this study, stress and pressure singularities induced by steady flows of viscous incompressible fluids are asymptotically identified. This is done by taking advantage of an earlier result that the NavierStokes equations are locally governed by Stokes flow in angular corners. Findings are confirmed by developing and using an analogy with solid mechanics, and by applying divergence checks to global numerical results. These flow-induced singularities render a number of structural configurations singular that were not previously appreciated as such from identifications within solid mechanics alone.
\end{abstract}

Keywords: incompressible viscous fluids, Navier-Stokes equations, flow-induced stress singularities.

\section{Introduction}

As an introductory example, we consider the steady two-dimensional flow of an incompressible viscous fluid over a step (fig. 1). The issue of concern is what stress singularities, if any, are induced by the fluid flow at the corners $C$ and $C^{\prime}$. Such singularities can in turn lead to stress singularities within the solid comprising the boundary. When a stress singularity occurs at location of concern within a structure, there are three approaches that may be adopted to try and ensure structural integrity: to dispense with any detailed stress analysis 


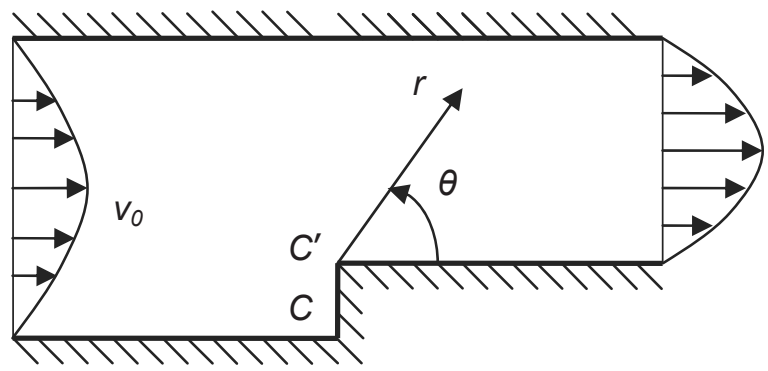

Figure 1: $\quad$ Flow over a step.

and rely on experimental testing; to apply a generalized fracture mechanics approach and take the coefficient of the singularity as the damage controlling parameter; or to improve the modeling so that the singularity is removed and physically-sensible stresses result. Essential to practicing any of the foregoing options is a recognition of the stress singularity's presence. Here our principal objective is to aid in this regard with respect to flow-induced stress singularities.

Although there is an extensive literature concerning the identification of stress singularities in solids in isolation (see Sinclair [1] for a review), the literature concerning the identification of stress singularities resulting from fluid-solid interactions is relatively sparse. There are a few implicit identifications that follow from the result shown in Kondrat'ev [2], and confirmed in Blum and Rannacher [3], that, asymptotically near a corner, the Navier-Stokes equations are controlled by Stokes flow. Beginning with Rayleigh [4], Stokes flow in corners has been considered by a number of investigators (e.g., Dean and Montagnon [5], Moffatt [6,7], Liu and Joseph [8]). While these investigations have primarily been concerned with elucidating Stokes flow fields, they do contain some eigenvalue equations that can be used in conjunction with $[2,3]$ to identify singularities. Here, then, we seek to carry out such singularity identifications more explicitly and for a broader class of fluid structure configurations than considered heretofore.

We begin in Section 2 with a local asymptotic analysis of steady twodimensional flows of incompressible viscous fluids in a wedge-shaped region subjected to a variety of boundary conditions. Though an effort is made to identify all of the flow-induced singularities for this class of fluid flows, there are going to be further configurations with such singularities. Accordingly, in Section 3, to guard against accepting finite values for pressures or stresses at singular points in such configurations, we offer some divergence checks for use in computational fluid dynamics. We demonstrate these checks on two global fluid structure configurations. We close, in Section 4, with some comments in light of the fluid structure stress singularities identified.

\section{Local asymptotic analysis}

The local problems of interest are for fluids within the wedge-shaped region $\mathscr{R}$. In terms of cylindrical polar coordinates $(r, \theta)$ (e.g., at $C^{\prime}$, fig. 1$)$, this region is 
defined by $\mathscr{R}=\{(r, \theta) \mid 0<r<\infty, 0<\theta<\phi\}$, where $\phi$ is the vertex angle within the fluid (e.g., $\phi=3 \pi / 2$ at $C^{\prime}$, fig. 1). For $\mathscr{R}, r$ is rendered dimensionless by normalization with a characteristic dimension. In general, we seek bounded fluid velocities $v_{r}, v_{\theta}$, with their companion pressure $p$ and stresses $\sigma_{r}, \sigma_{\theta}$ and $\tau_{r \theta}$, satisfying the governing field equations for steady, viscous, incompressible flow of a Newtonian fluid throughout $\mathscr{h}$, as well as boundary conditions on the edges of $\mathscr{A}$ : In particular, we seek the pressure and stresses near the vertex of $\mathscr{h}$ as $r$ $\rightarrow 0$. Here velocities are rendered dimensionless by normalization with a characteristic velocity, and the pressure and stresses are rendered dimensionless by normalization with a corresponding dynamic pressure.

More precisely, the governing field equations are: the equation of continuity for an incompressible fluid,

$$
\frac{\partial}{\partial r}\left(r v_{r}\right)+\frac{\partial v_{\theta}}{\partial \theta}=0
$$

on ; the Navier-Stokes equations for steady motion of an incompressible fluid,

$$
\begin{gathered}
D v_{r}-\frac{v_{\theta}^{2}}{r}=-\frac{\partial p}{\partial r}+\frac{1}{R e}\left(\nabla^{2} v_{r}-\frac{v_{r}}{r^{2}}-\frac{2}{r^{2}} \frac{\partial v_{\theta}}{\partial \theta}\right), \\
D v_{\theta}+\frac{v_{r} v_{\theta}}{r}=-\frac{1}{r} \frac{\partial p}{\partial \theta}+\frac{1}{R e}\left(\nabla^{2} v_{\theta}+\frac{2}{r^{2}} \frac{\partial v_{r}}{\partial \theta}-\frac{v_{\theta}}{r^{2}}\right),
\end{gathered}
$$

on $\mathscr{h}$, where $R e$ is Reynold's number, and $D=v_{r} \partial / \partial r+v_{\theta} r^{-1} \partial / \partial \theta$; and the stress-velocity relations for an incompressible Newtonian fluid,

$$
\left\{\begin{array}{c}
\sigma_{r} \\
\sigma_{\theta}
\end{array}\right\}=-p\left\{\begin{array}{l}
+ \\
-
\end{array}\right\} \frac{2}{R e} \frac{\partial v_{r}}{\partial r}, \tau_{r \theta}=\frac{1}{R e}\left(\frac{1}{r} \frac{\partial v_{r}}{\partial \theta}+\frac{\partial v_{\theta}}{\partial r}-\frac{v_{\theta}}{r}\right),
$$

on भ. The boundary conditions are any one of the admissible sets listed in table 1 on the boundary of the fluid at $\theta=0$, together with another such set on the boundary at $\theta=\phi$, for $0<r<\infty$. Conditions I of table 1 are the traditional no slip conditions for a fluid in contact with a solid. Conditions II are Maxwell's relaxation of I to permit some fluid flow parallel to a solid boundary: In these conditions, $c$ is a constant. These two sets of conditions occur either in concert with themselves or with Conditions III on the boundaries of $\mathscr{R}$.

Analysis of the foregoing asymptotic problems proceeds on introducing a stream function $\psi$, then seeking $\psi$ as the sum of a series of separable solutions with increasing powers of $r$. That is, we take

$$
v_{r}=\frac{-1}{r} \frac{\partial \psi}{\partial \theta}, v_{\theta}=\frac{\partial \psi}{\partial r}, \psi=r^{\lambda+1} f_{\lambda}+r^{\lambda^{i+1}} g_{\lambda}+\ldots,
$$

wherein $\lambda$ and $\lambda^{\prime}$ are constants while $f_{\lambda}$ and $g_{\lambda^{\prime}}$ are functions of $\theta$. Then eqn (1) is satisfied. Bounded velocities require that $\lambda \geq 0$ and $\lambda^{\prime} \geq 0$ : without loss of generality, we order these constants so that $0 \leq \lambda<\lambda^{\prime}$. With this ordering, the dominant terms on introducing eqns (4) into eqns (2) are:

$$
O\left(r^{2 \lambda-1}\right)=O\left(r^{\lambda-2}\right)+O\left(R e^{-1} r^{\lambda-2}\right) \text { as } r \rightarrow 0,
$$


on $\mathscr{R}$. The dominant terms within eqn (5) thus are those on the right-hand side. These are set to zero by themselves. Thereafter, the term on the left-hand side can interact with the dominant terms for $\lambda^{\prime}$ on taking $\lambda^{\prime}=2 \lambda+1$, then the terms on the left-hand side for $\lambda^{\prime}$ can interact with yet higher powers of $r$, and so on. As a result, the problem for the $\lambda$-fields is reduced to the linear equations for Stokes flow (this outcome is more formally developed in [2,3]).

Table 1: $\quad$ Boundary conditions.

\begin{tabular}{ccc}
\hline Roman numeral & Conditions & Physical description \\
\hline I & $v_{r}=v_{\theta}=0$ & No slip \\
II & $v_{r}=\frac{c}{r} \frac{\partial v_{r}}{\partial \theta}, v_{\theta}=0$ & Maxwell slip \\
III & $\sigma_{\theta}=\tau_{r \theta}=0$ & Free surface \\
\hline
\end{tabular}

For Stokes flow, eliminating the pressure terms from the so-reduced NavierStokes equations establishes that $\psi$ is biharmonic. Hence we take the known solution

$$
\psi=r^{\lambda+1}\left[c_{1} \cos (\lambda+1) \theta+c_{2} \sin (\lambda+1) \theta+c_{3} \cos (\lambda-1) \theta+c_{4} \sin (\lambda-1) \theta\right],
$$

where $c_{i}, i=1-4$, are constants. Developing corresponding fields from eqns (4), (2) and (3) and substituting into any of the admissible combinations of boundary conditions of table 1 leads to four homogeneous equations in the four constants $c_{i}$. A nontrivial solution requires that the determinant of this system be zero and so generates the corresponding eigenvalue equation for $\lambda$ for the boundary conditions involved. These equations are set out in table 2 .

Table 2: $\quad$ Eigenvalue equations.

\begin{tabular}{ccc}
\hline $\begin{array}{c}\text { Boundary } \\
\text { conditions }\end{array}$ & Equation & Physical description \\
\hline I-I & $\sin ^{2} \lambda \phi=\lambda^{2} \sin ^{2} \phi$ & No slip - no slip \\
II-II & $\cos ^{2} \lambda \phi=\cos ^{2} \phi$ & Maxwell slip - Maxwell slip \\
I-III & $\cos ^{2} \lambda \phi=\lambda^{2} \sin ^{2} \phi$ & No slip - free \\
II-III & $\sin 2 \lambda \phi=-\lambda \sin 2 \phi$ & Maxwell slip - free \\
\hline
\end{tabular}

From eqns (4), (2) and (3), 


$$
\sigma=O\left(r^{-\gamma}\right) \text { as } r \rightarrow 0, \gamma=1-\lambda,
$$

where $\sigma$ denotes any stress component or the pressure. Then for $0 \leq \lambda<1, \gamma>0$ and $\gamma$ is the singularity exponent for the stresses and pressure. Solving the eigenvalue equations in table 2 for $\lambda$ in this range thus leads to the singularity exponents for power singularities displayed in fig. 2.

In fig. $2, \Phi$ is the angle exterior to the fluid at the vertex of $\mathscr{h}$, and hence the angle within the solid bounding the fluid. That is

$$
\Phi=2 \pi-\phi .
$$

A few of the singularity exponents shown in fig. 2(a) are effectively given in $[5,7]$ : Whenever this occurs, the values in fig. 2(a) are in agreement. We can now answer the question posed in the Introduction. For $C$ of fig. $1, \Phi=270^{\circ}$ and fig. 2(a) has that there are no flow-induced singularities. In contrast, for $C^{\prime}$ of fig. $1, \Phi=90^{\circ}$ and fig. 2(a) has that there are two flow-induced singularities.

Comparing Maxwell's slip with no slip conditions, figs. 2(a) and (b) indicate that Maxwell's slip has more singularities and stronger singularities, but singularities over the same range of exterior vertex angles, namely $\Phi<180^{\circ}$. With mixed conditions I - III, fig. 2(c) shows that up to four different singularities can occur for a single $\Phi$, and that the range of $\Phi$ with singularities is extended up to $\Phi<315^{\circ}$. With mixed conditions II-III, fig. 2(d) shows that up to three different singularities can occur for a single $\Phi$ for a range of $\Phi<270^{\circ}$, again a larger range than for non-mixed conditions.

The eigenvalues underlying the singularity exponents in fig. 2 can be further confirmed by comparison with eigenvalues from plane strain elasticity when the elastic solid is taken to be incompressible. The field theory for Stokes flow is analogous to that for incompressible elasticity if velocity components are exchanged for corresponding displacements. Under this exchange, no slip conditions are analogous to clamped conditions and Maxwell slip conditions to frictionless contact conditions, while free surface conditions remain as themselves. Then exactly the same eigenvalue equations as in table 2 occur for analogous conditions in plane strain incompressible elasticity (see [1]). Moreover, resulting eigenvalues can be confirmed by comparison with the extensive set of eigenvalues provided in Seweryn and Molski [9]. When this is done, complete agreement is found to within the significant figures available.

This analogy has the additional benefit of helping to identify log singularities. That is,

$$
\sigma=O(\ln r) \text { as } r \rightarrow 0
$$

Some effort has been made to identify log singularities in elasticity. The configurations so found are given in [1]. Using the analogy, table 3 sets out configurations with flow-induced log singularities.

In table 3, $\omega$ is an angular velocity so that Conditions $\mathrm{I}_{\omega}^{+}$with $\mathrm{I}_{\omega}^{-}$effect a pinching of the fluid with no slip while $\mathrm{II}_{\omega}^{+}$with $\mathrm{II}_{\omega}^{-}$effect a pinching with Maxwell slip. Also, Conditions $\mathrm{III}_{\tau}$ apply a constant shear traction, $\tau$ : 


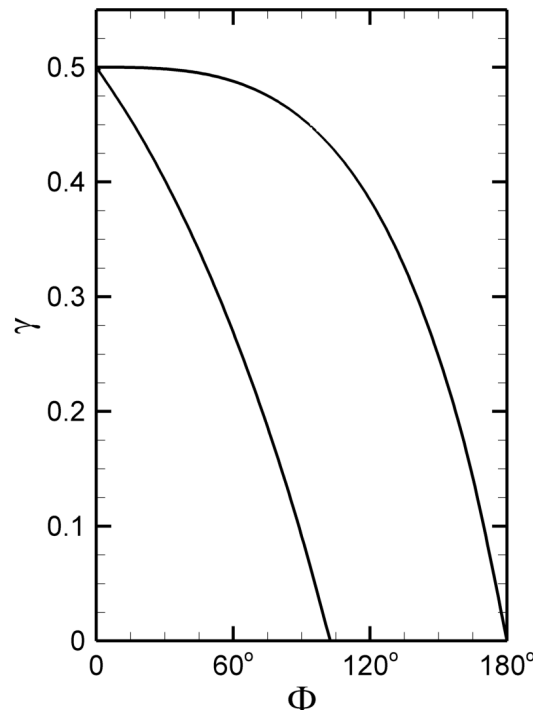

(a)

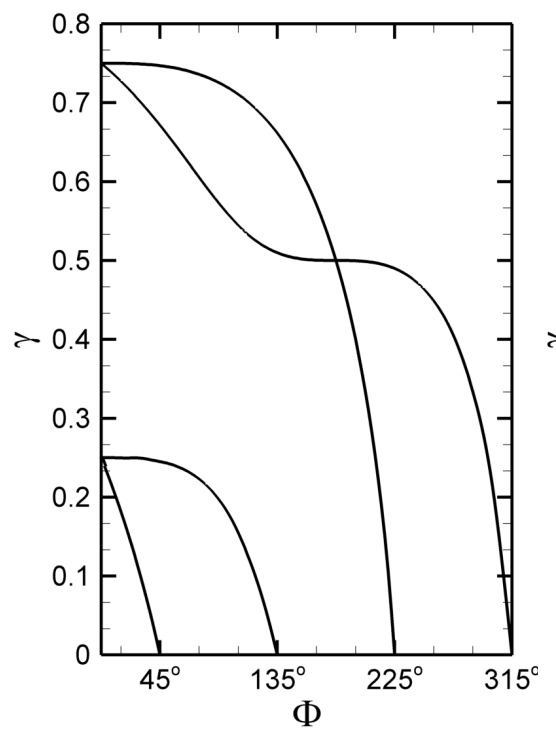

(c)

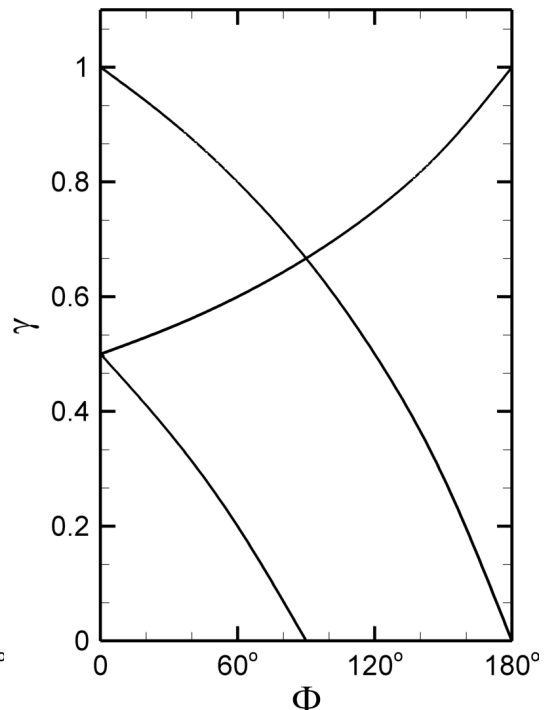

(b)

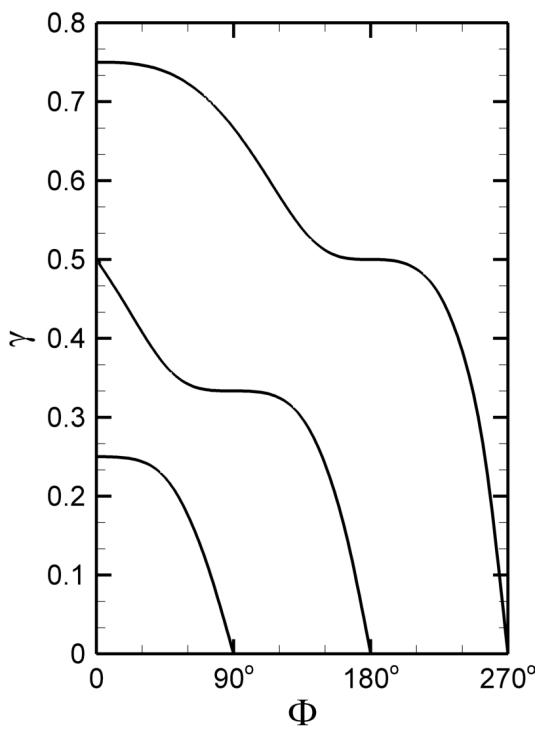

(d)

Figure 2: $\quad$ Singularity exponents - (a) for no slip and no slip, (b) for Maxwell slip and Maxwell slip, (c) for no slip and free, and (d) for Maxwell slip and free. 
Although this does not appeal much as a physically applicable condition for fluids, it has been entertained in the literature (see [6]). Complete fields containing logarithmic singularities in the stresses and pressures can be generated from an auxiliary stream function formed by differentiating eqn (6) with respect to $\lambda$.

Table 3: Log singularities.

Modified boundary conditions Configurations with log singularities

$$
\left(\phi^{*}=\tan \phi^{*} ; n=1,3\right)
$$

$$
\begin{array}{cc}
\mathrm{I}_{\omega}^{+}: v_{r}=0, v_{\theta}=\omega r & \mathrm{I}-\mathrm{I}, \phi=\phi^{*} \\
\mathrm{I}_{\omega}^{-}: v_{r}=0, v_{\theta}=-\omega r & \mathrm{I}_{\omega}^{+}-\mathrm{I}_{\omega}^{-} \\
\mathrm{II}_{\omega}^{+}: v_{r}=\frac{c}{r} \frac{\partial v_{r}}{\partial \theta}, v_{\theta}=\omega r & \mathrm{II}_{\omega}^{+}-\mathrm{II}_{\omega}^{-} \\
\mathrm{II}_{\omega}^{-}: v_{r}=\frac{c}{r} \frac{\partial v_{r}}{\partial \theta}, v_{\theta}=-\omega r & \mathrm{I}-\mathrm{III}_{\tau}, \phi=(2 n \pm 1) \pi / 4 \\
\mathrm{III}_{\tau}: \sigma_{\theta}=0, \tau_{r \theta}=\tau & \mathrm{II}-\mathrm{III}_{\tau}, \phi=n \pi / 2
\end{array}
$$

\section{Global numerical analysis}

Armed with an asymptotic appreciation of the presence of a flow-induced stress singularity in a fluid structure configuration, the futile exercise of attempting to compute stresses at the singularity can be avoided. However, there are flowinduced singularities that have yet to be asymptotically identified, and possibly some such configurations that are not even suspected of being singular. In such instances, convergence-divergence checks may serve to guard against accepting the necessarily finite values of stresses and pressures that result from global numerical analysis when in fact the stresses and pressure are singular.

In order to do this, convergence-divergence checks need to reject numerical values when either power or log singularities occur. Because log singularities are weaker than power, the greater challenge for checks in this regard is the rejection of numerical values for log singularities.

At the outset in designing convergence-divergence checks, we look to evaluate their parallel performance on converging or diverging series because series afford a ready one-dimensional assessment. With log singularities in mind, we focus on the harmonic progression because it is logarithmically divergent. For the harmonic progression, partial sums are 


$$
S_{N}=\sum_{n=1}^{N} \frac{1}{n} \sim \ln (N+1 / 2)+C_{E}+O\left(N^{-2}\right) \text { as } N \rightarrow \infty,
$$

where $C_{E}$ is Euler's constant.

It is sometimes suggested in the literature that convergence can be assessed by comparing results when the number of cells in a discretization increases linearly. For series, this corresponds to comparing differences in partial sums for $N, 2 N, 3 N, \ldots, m N,(m+1) N, \ldots$,

with convergence being predicted when differences between successive partial sums decrease and eventually become sufficiently small. Alternatively, it is suggested that convergence can be assessed by comparing results when the number of cells in a discretization increases by a fixed factor. For series, this corresponds to comparing differences in partial sums for

$$
N, \alpha N, \alpha^{2} N, \ldots, \alpha^{m-1} N, \alpha^{m} N, \ldots
$$

wherein $\alpha$ is the fixed factor, with convergence again being predicted when differences between successive partial sums decrease and eventually become sufficiently small. For either of these approaches to be valid, we need it to not conclude that the harmonic progression is converging.

Applying the sequence of (11) to eqn (10) yields

$$
\Delta S_{m+1}=S_{(m+1) N}-S_{m N} \sim \ln \left(1+m^{-1}\right)+O\left(m^{-2}\right) \text { as } m \rightarrow \infty .
$$

Hence differences decrease to zero and convergence is erroneously predicted. Applying the sequence of (12) to eqn (10) yields

$$
\Delta S_{m+1}=S_{\alpha^{m} N}-S_{\alpha^{m-1} N} \sim \ln \alpha+O\left(\alpha^{-m}\right) \text { as } m \rightarrow \infty .
$$

Now differences approach a constant, and so are not decreasing and convergence is not predicted. We therefore adopt the sequence of (12) as underlying our mesh refinement in convergence-divergence checks.

What the foregoing series simulation indicates is that we can expect a log singularity to be revealed by successive changes in the stress of concern approaching a constant when the number of cells successively increases by a fixed factor. To implement convergence-divergence checks to take advantage of this singularity signature, we need to decide what constitutes being a "constant". Absent any fluid, a 10\% rule has been demonstrated to be effective in stress analysis (see Sinclair et al. [10]). Accordingly we adopt a 10\% rule here.

More precisely, we now let $N$ denote the number of cells used in the numerical analysis and $m$ denote the mesh number with a number of cells as given by the sequence of (12). We take $\alpha=4$ as our fixed factor corresponding to halving cell sides in two dimensions. This choice is merely easy to implement, at least initially: Other choices are possible. We designate the stress of concern as computed on mesh $m$ by $\sigma_{m}$, and define the increment in the value of this stress attending refinement to produce mesh $m$ by $\Delta \sigma_{m}=\sigma_{m}-\sigma_{m-1}$. Then for such stress increments,

$$
\begin{gathered}
\left|\Delta \sigma_{m-1}\right|>1.1\left|\Delta \sigma_{m}\right| \Rightarrow \text { converging, } \\
\left|\Delta \sigma_{m-1}\right| \leq 1.1\left|\Delta \sigma_{m}\right| \Rightarrow \text { divergence }
\end{gathered}
$$

with divergence indicating the possibility of a stress singularity being present. 
As a first application of the foregoing convergence-divergence checks, we return to the global problem of flow in a channel with a forward-facing step on the bottom wall (fig. 1). The channel height upstream of the step is $40 \mathrm{~mm}$, and the step height is $10 \mathrm{~mm}\left(C-C^{\prime}\right.$ in fig. 1). The distance from the inlet to the step and the distance from the step to the channel exit are both $100 \mathrm{~mm}$. No slip boundary conditions are applied on all channel walls. The fluid entering the channel has a maximum velocity $v_{0}=0.5 \mathrm{~m} / \mathrm{s}$, and its density and viscosity are taken such that $R e=1370$ based on the upstream channel height.

This Reynolds number is well below the value of 2300 for transition flows. Hence the flow in the channel can be taken to be laminar. The FLUENT code is used to compute solutions, with the specific algorithm selected being SIMPLE with second-order upwind differencing for the momentum transport.

An initial mesh $(m=1)$ is taken with $N=7,000$ square cells of extent $1 / 10$ of the height of the step. This mesh is systematically refined throughout by halving cell sides $(\alpha=4)$ to furnish meshes $m=2$ and 3 with 28,000 and 112,000 cells, respectively. Results for the pressure coefficient, $C_{p}$, at both corners $\left(C\right.$ and $C^{\prime}$, fig. 1) are included in table 4.

Table 4: $\quad$ Pressure coefficients from successively refined meshes.

\begin{tabular}{ccccc}
\hline$m$ & $C_{p} @ C$ & $C_{p} @ C^{\prime}$ & $C_{p} @ U$ & $C_{p} @ V$ \\
\hline 1 & 1.5509 & 0.0454 & 3.8209 & 4.0044 \\
2 & 1.5605 & -0.2565 & 3.8283 & 4.0487 \\
3 & 1.5606 & -0.7198 & 3.8301 & 4.0850 \\
4 & - & - & 3.8305 & 4.1183 \\
\hline
\end{tabular}

In table 4, pressure coefficients at $C$ are in accord with criterion (15) and therefore judged to be converging. Indeed, for $m=3, C_{p}$ appear to have converged to within $0.01 \%$. This is as it should be, there being no singularity at $C$. On the other hand in table 4, pressure coefficients at $C^{\prime}$ are in accord with criterion (16) and therefore judged to exhibit divergence. This is also as it should be, there being two power singularities at $C^{\prime}(\gamma=0.46,0.09$, fig. 2(a)).

While the divergence check of eqn (16) works satisfactorily on this first global problem, this problem does have a strong singularity thereby facilitating numerical detection. To provide a more stringent test of these convergencedivergence checks, we next consider their application to a global problem with but a weak log singularity.

This global problem is for the symmetric flow in a channel past a wall with a forward-facing, single, sharp, knife edge (fig. 3, just the upper half of the flow path shown). The total channel height upstream is now $80 \mathrm{~mm}$ (by reflection about the $x$ axis of the $40 \mathrm{~mm}$ height shown in fig. 3). The height, $h$, of the half of the central wall shown in fig. 3 is given by 


$$
\frac{h}{L}=\frac{1}{8} \tan \frac{\phi^{*}}{2}\left[\left(1-\frac{x}{L}\right)^{8}-1\right],
$$

for $0 \leq x \leq L$, where $L=100 \mathrm{~mm}$ is the length from the knife edge vertex, $V$, to the channel exit. The length from the channel entrance to $V$ is also $L$. No slip boundary conditions are applied on all solid boundaries, and symmetry conditions are applied on $-L \leq x \leq 0, y=0$ (fig. 3). Then when $\phi^{*}$ is as in table 3 , the log singularity at the top of table 3 is realized.

The same fluid properties as earlier are used, but now the maximum velocity at the channel entrance is taken to be $0.25 \mathrm{~m} / \mathrm{s}$ to compensate for doubling the total inlet height and so keep $R e$ in the laminar range.

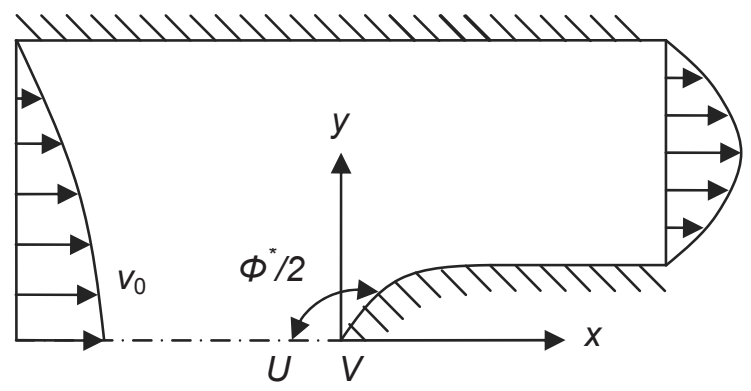

Figure 3: $\quad$ Flow past a knife-edged wall.

The same FLUENT algorithm as previously is employed, but now on a sequence of four meshes, $m=1-4$. The initial structured mesh has 32,000 cells. Subsequent meshes are systematically refined throughout and have cell numbers that successively increase by a factor of four (thus $N \doteqdot 2$ million for $m=4$ ). Results for $C_{p}$ at $V$ as well as at $U, L / 5000$ upstream of $V$, are included in table 4 .

In table 4 , pressure coefficients at $U$ are in accord with criterion (15) and therefore judged to be converging. Indeed these $C_{p}$ appear to enjoy a quadratic convergence rate and to have converged within $0.01 \%$. This is as it should be, there being no singularity upstream on the line of symmetry. Unfortunately in table 4 , pressure coefficients at $V$ initially $(m=1,2,3)$ are also in accord with criterion (15), and converging predicted despite the presence of a log singularity. This erroneous prediction is the result of the convergence of other nonsingular contributions at $V$ that have decreasing $C_{p}$ increments that occur in concert with the constant increments caused by the log singularity. Ultimately with mesh refinement, this disguising of constant increments has to cease, and this starts to happen here on meshes $m=2,3,4$. For these meshes, criterion (16) is complied with and divergence appropriately predicted. This global problem, though, does serve notice of the risk that weak log singularities can go undetected with convergence-divergence checks. 


\section{Concluding remarks}

Flow-induced power and log singularities in stresses and pressures are asymptotically identified in this study for a range of configurations with steady, incompressible, viscous flows. These findings are confirmed by an analogy with stress singularities in incompressible elastic solids. They are further confirmed to some extent by global numerical analysis on a sequence of appropriately refined meshes. The convergence-divergence checks used to effect this second confirmation can also be used as an alternative means of identifying singularities provided mesh refinement is continued to a sufficient extent.

These flow-induced singularities occur for distinctly different configurations than those for structures devoid of any fluid interaction despite the two being analogous. By way of example, the corner at $C$ in fig. 1 does have a singularity in elasticity while the corner at $C^{\prime}$ does not. In contrast, there is no flow-induced singularity at $C$ but there is a flow-induced singularity at $C^{\prime}$.

The fluid structure interactions considered here depart from the norm in which both the fluid mechanics and the solid mechanics have to be analyzed simultaneously. Rather here the fluid mechanics can be treated separately first, and then, in effect, it sets boundary conditions for the subsequent solid mechanics. Nonetheless it is essential to take into account the interaction between the two and recognize any flow-induced singularities so produced if any attempt is to be made to try and ensure structural integrity at the locations of such singularities.

\section{References}

[1] Sinclair, G.B., Stress singularities in classical elasticity - II: Asymptotic identification. Applied Mechanics Reviews, 57, pp. 385-439, 2004.

[2] Kondrat'ev, V.A., Asymptotic solution of the Navier-Stokes equations near the angular point of the boundary. Journal of Applied Mathematics and Mechanics, 31, pp. 125-129, 1967.

[3] Blum, H. \& Rannacher, R., On the boundary value problem of the biharmonic operator on domains with angular corners. Mathematical Methods in the Applied Sciences, 2, pp. 556-581, 1980.

[4] Rayleigh, Lord, Scientific Papers VI, Cambridge University Press: Cambridge, pp. 18-21, 1920.

[5] Dean, W.R. \& Montagnon, P.E., On the steady motion of viscous liquid in a corner. Proceedings of the Cambridge Philosophical Society, 45, pp. 389-394, 1949.

[6] Moffatt, H.K., Viscous and resistive eddies near a sharp corner. Journal of Fluid Mechanics, 18, pp. 1-18, 1964.

[7] Moffatt, H.K., The asymptotic behaviour of solutions of the Navier-Stokes equations near sharp corners. Proc. of the Symp. on Approximation Methods for Navier-Stokes Problems, Springer Verlag: Berlin, pp. 371$380,1979$. 
[8] Liu, C.H. \& Joseph, D.D., Stokes flow in wedge-shaped trenches. Journal of Fluid Mechanics, 80, pp. 443-463, 1977.

[9] Seweryn, A. \& Molski, K., Elastic stress singularities and corresponding generalized stress intensity factors for angular corners under various boundary conditions. Engineering Fracture Mechanics, 55, pp. 529-556, 1996.

[10] Sinclair, G.B., Beisheim, J.R. \& Sezer, S., Practical convergencedivergence checks for stresses from FEA. Proc. of the 2006 International ANSYS Conf., Pittsburgh, on CD-ROM, 2006 (copy available from sinclair@me.lsu.edu). 